\title{
Mathematical Modeling of Creep in a Functionally Graded Rotating Disc with Varying Thickness
}

\author{
Vandana Gupta $^{1} \cdot$ S. B. Singh ${ }^{1}$
}

Received: 25 June 2015 / Accepted: 26 July 2016 / Published online: 19 August 2016

(C) The Regenerative Engineering Society 2016

\begin{abstract}
This study puts forward an analytical framework for the analysis of creep stresses and creep rates in the isotropic rotating nonFGM/FGM disc with uniform and varying thickness. The material parameters of creep vary along the radial distance in the disc due to varying composition, and this variation has been estimated by regression fit of the available experimental data. The creep behavior of the disc under stresses developing due to rotation has been determined following Sherby's law. The creep response of rotating disc is expressed by threshold stress with value of stress exponent as 8 . The results obtained for isotropic non-FGM/FGM constant thickness disc have been compared to those estimated for isotropic varying thickness disc with the same average particle content distributed uniformly. Overall, our results suggest that the distribution of stresses and strain rates becomes relatively more uniform in the isotropic FGM hyperbolic thickness disc.
\end{abstract}

\section{Lay Summary}

The purpose of this paper is to investigate the steady-state creep response in the isotropic non-FGM/FGM varying thickness disc with linear variation of particle distribution along the radial distance. Mathematical model to describe steady-state creep behavior in an isotropic rotating disc made of silicon carbide particulate composite has been developed. Three variations in the thickness (constant, linear, and hyperbolic) of the rotating composite non-FGM/FGM disc have been

Vandana Gupta

vaggarwal2584@gmail.com

S. B. Singh

sbsingh69@yahoo.com

1 Department of Mathematics, Punjabi University, Patiala 147002, Punjab, India considered, while keeping average volume of all the discs is the same. The study revealed that the strain rates are the lowest and more uniform in the FGM hyperbolic thickness disc having reinforcement distributed in a linear way as compared to non-FGM/FGM discs with constant and linear thickness.

Keywords Modeling $\cdot$ Composites $\cdot$ Rotating disc $\cdot$ Creep · Functionally graded material (FGM)

\section{Introduction}

Functionally graded materials (FGMs) have been proved to be attractive materials for a broad range of engineering applications, tissue engineering, or regenerative medicine. The body of every living being contains bones and teeth as natural functionally graded materials. In the same way, artificial tissues in orthopedic applications are made of functionally graded materials. Loading conditions of a particular tissue decide the tailoring methodology of that tissue. The strengthening of an artificial tissue is done accordingly to replace a natural tissue in living beings. The biocompatibility of these tissues depends on their mechanical properties. In this paper, mechanical properties of FGM in a disc have been investigated.

On the other side, in engineering applications (automobiles, aircraft, sports, aero-engines, rotors, turbines, pumps, compressors, flywheels, braking systems of automotive), the disc is subjected to severe mechanical loads and is also exposed to elevated temperatures. Under these high-temperature applications, the conventional materials (metals/ceramics) may not survive alone. Thus, a concept of functionally graded material was introduced and led to the development of superior heat-resistant materials. The objective of developing the FGM concept is that the properties of the materials will be improved and new functions for them established. Wahl et al. [23] were the first to investigate 
theoretically steady-state creep behavior by a power function in a rotating turbine disc made of chromium steel using von Mises and Tresca yield criteria theoretically describing creep behavior and compared the results with experimental values. Ma [10] derived some formulas based on the maximum shear theory associated with the von Mises flow rule for calculating creep deformations and stress distributions in rotating disc of variable thickness at uniform temperature by using the power function for creep behavior. The results obtained using von Mises criterion are found to be in excellent agreement with the available experimental creep data [23]. Ma [11, 12] investigated the stress analysis of rotating solid discs having variable thickness and operating under a temperature gradient in the radial direction of the disc. The analysis was based on the theory of Tresca's criterion. Bhatnagar et al. [3] have studied creep behavior of orthotropic rotating discs having variable thickness (constant, linear, and hyperbolic) using Norton's power law and concluded that by selecting a certain type of material anisotropy and an optimum profile for the disc, the creep rate in the rotating disc can be reduced. Mishra and Pandey [13] proposed that steady-state creep in aluminum-based composites could be described in a better way by Sherby's constitutive creep model, as compared to Norton's creep model. Pandey et al. [14] have studied the steady-state creep behavior of Al-SiCp composites under uniaxial loading condition in the temperature range between 623 and $723 \mathrm{~K}$ for different combinations of particle size and volume fraction of reinforcement and found that the composite with finer particle size has better creep resistance than that containing coarser ones. Penny and Mariott [15] investigated the creep behavior of a thin rotating disc by proposing a method for multiaxial creep calculation of a rotating disc using the fundamental solution for creep of a uniform disc and extended it to non-uniform discs/ cylinders under different loading conditions. Reddy et al. [18] studied functionally graded solid and annular circular discs with a mixture of ceramic and metals and concluded that the ceramic constituent of the material provides high-temperature resistance due to its low thermal conductivity. Singh and co-workers [4, 7, $8,16,17,21]$ have extensively studied creep in isotropic/ anisotropic rotating discs during last two decades. Authors have determined creep in isotropic, anisotropic, FGM discs of constant thickness. Jahed et al. [9] concluded that the use of variable thickness disc helps in minimizing the weight of disc in aerospace applications, and minimizing the weight of such items in aerospace applications gives advantages such as low dead weights and lower costs. Zenkour [24] developed an analytical solution for elastic deformation of the rotating functionally graded disc and investigated the effect of material properties on the distributions of the displacement and stresses along the radial direction of the disc. Sharma and Sahni [19] derived elastic-plastic and transitional stresses in a variable thickness disc having inclusion by using transition theory and observed that the rotating disc made of incompressible material with inclusion requires higher angular speed to yield at the internal surface as compared to disc made of compressible material. It is concluded that the disc made of isotropic compressible material is on the safer side of design as compared to disc made of isotropic incompressible material as it requires higher percentage increase in angular speed to become fully plastic from its initial yielding. Bayat et al. [1] carried out thermo-elastic analysis for axisymmetric rotating discs made of functionally graded material (FGM) with variable thickness by using small and large deflection theory and noticed that mechanical responses in rotating disc made of functionally graded material can be smaller than observed in a homogeneous disc for particular values of grading index of material properties. Bektas and Acka [2] investigated the stress analysis of functionally graded discs subjected to internal pressure and various temperature distributions. They obtained closed form solutions for stresses and displacements by using the infinitesimal deformation theory of elasticity. The results obtained both analytical and numerical are found very well consistent with each other. Deepak et al. [5] investigated the effect of varying disc thickness gradient on creep response of functionally graded rotating discs with linearly varying thickness. It can be concluded that the stresses and strain rates in both the radial and tangential directions reduce significantly with the increase in thickness gradient of the composite disc. Garg et al. [6] carried out the steady-state creep analysis in linearly varying thickness rotating disc made of functionally graded composite and with stress exponent 5 . The study reveals that the magnitude of strain rates and extent of distortion in the disc could be reduced significantly by employing FGM linearly varying thickness disc with higher reinforcement.

Keeping this in mind, it may be interesting to extend the above analysis for rotating isotropic non-FGM/FGM disc with variable thickness. Emphasis has been done on development of analytical models capable of performing plastic stress and strain analyses for rotating isotropic non-FGM/FGM discs. The content of silicon carbide particles in the discs is assumed to decrease linearly from the inner radius to the outer radius of the disc. The creep response in composite disc rotating at 15,000 rpm has been analyzed by Sherby's constitutive model and von Mises criteria for yielding.

\section{Assumptions in Variable Thickness Disc and Disc Profile}

We consider an aluminum silicon carbide particulate composite disc of constant thickness $h$ having inner radius $a$, outer radius $b$, and rotating with angular velocity, $\omega$ (radian/s). For the purpose of creep analysis in disc with variable thickness, the following assumptions are made:

1. Material of disc is incompressible and locally isotropic, i.e., the properties of the disc remain constant at a given radius in all the directions but can change with the change in radius. 
2. Stresses at radius of the disc remain constant with time, i.e., steady-state condition of stress is assumed.

3. Elastic deformations are small for the disc, and therefore, they can be neglected as compared to creep deformation.

4. Axial stress in the disc may be assumed to be zero as thickness of disc is assumed to be very small compared to its diameter.

5. Frictional shear stress induced due to braking action is estimated to be $10^{-5} \mathrm{MPa}$, which is very small compared to creep stresses and therefore can be neglected.

6. The composite shows a steady-state creep behavior, which may be described by following Sherby's law [20]:

$\dot{\bar{\varepsilon}}=\left[M(r)\left(\bar{\sigma}-\sigma_{0}(r)\right)\right]^{n}$,

where

$M(r)=\frac{1}{E}\left(\frac{A D_{D} \lambda^{3}}{\left|b_{r}\right|^{5}}\right)^{1 / n}$ is the creep parameter, where $\dot{\bar{\varepsilon}}$, $\bar{\sigma}, n, \sigma_{0}(r), A, D_{\lambda}, \lambda, b_{r}$, and $E$ are the effective strain rate, effective stress, the stress exponent, threshold stress, a constant, lattice diffusivity, the sub grain size, the magnitude of burgers vector, and Young's modulus.

In particle-reinforced composite, the material parameters $M(r)$ and $\sigma_{0}(r)$ depend on the particle size and the percentage of dispersed particles apart from the temperature, which have been obtained by using the experimental results of Pandey et al. [14] (Table 1). The following regression equations have been extracted from the available experimental results by using DATAFIT software.

$M(r)=e^{-35.38} P^{0.2077} T^{4.98} V(r)^{-0.622}$

$\sigma_{0}(r)=-0.03507 P+0.01057 T+1.00536 V(r)-2.11916$

In a FGM disc, both the creep parameters $M(r)$ and $\sigma_{0}(r)$ will vary due to variation with particle content, and both are functions of radial distance, which could be determined by substituting the values of particle and temperature, respectively.

Let $I$ and $I_{0}$ denote the moment of inertia of the disc at inner radius $a$ and outer radius $r$ and $b$, respectively. $A$ and $A_{0}$ denote the area of cross section of disc at inner radius $a$ and outer radius $r$ and $b$, respectively. Then,

$$
\begin{gathered}
I=\int_{a}^{r} h r^{2} d r, \quad I_{0}=\int_{a}^{b} h r^{2} d r \\
A=\int_{a}^{r} h d r, \quad A_{0}=\int_{a}^{b} h d r,
\end{gathered}
$$

Table 1 Creep parameters based on the experimental results [14]

\begin{tabular}{lllll}
\hline $\begin{array}{l}\text { Particle size } \\
P(\mu \mathrm{m})\end{array}$ & Temperature & Particle content & $M$ & $\sigma_{0}$ \\
1.7 & $T(\mathrm{~K})$ & $V(\mathrm{vol} \%)$ & $\left(\mathrm{s}^{-1 / 8} / \mathrm{MPa}\right)$ & $(\mathrm{MPa})$ \\
14.5 & & & 0.00963 & 15.24 \\
45.9 & 623 & 10 & 0.01444 & 11.46 \\
& & & 0.01897 & 13.65 \\
1.7 & 623 & 20 & 0.00963 & 15.24 \\
& & 30 & 0.00594 & 24.83 \\
& 623 & & 0.00518 & 34.32 \\
1.7 & 673 & 20 & 0.00594 & 24.83 \\
& 723 & & 0.00897 & 24.74 \\
& & & 0.01295 & 25.72 \\
\hline
\end{tabular}

where the average tangential stress may be defined as

$\sigma_{\theta \text { avg }}=\frac{1}{A_{0}} \int_{a}^{b} h \sigma_{\theta} d r$.

\section{Dises with Linearly Varying Thickness}

The thickness $(h)$ of the composite disc having linearly varying thickness is assumed to be of the form

$h=h_{b}+2 c(b-r)$,

where $c=\frac{\left(h_{a}-h_{b}\right)}{2(b-a)}$ is the slope of a disc, and $h_{a}$ and $h_{b}$ are the disc thickness at the inner and outer radii, respectively (Fig. 1).

Since the volume of disc having linearly varying thickness is assumed equal to the volume of constant thickness disc, therefore,

$$
\int_{a}^{b} 2 \pi r h d r=\pi\left(b^{2}-a^{2}\right) t
$$

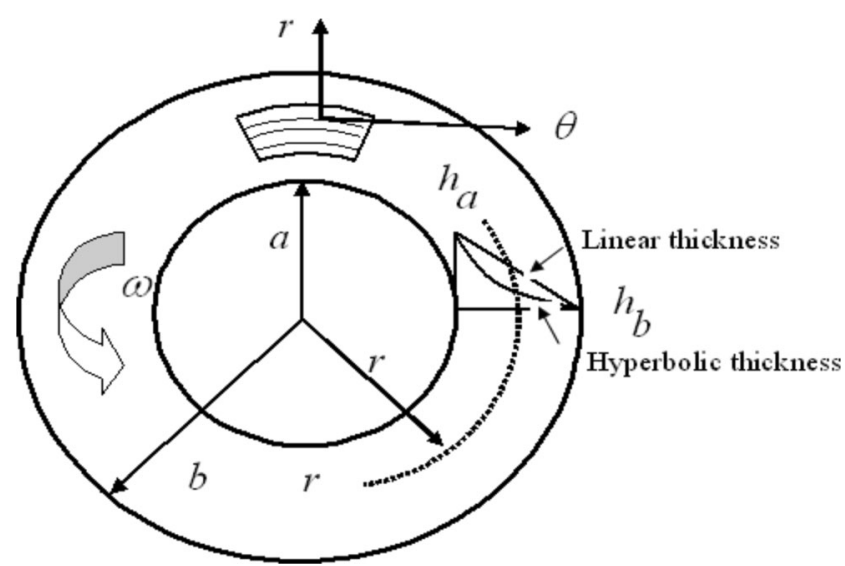

Fig. 1 Geometry of rotating disc by showing variation in thickness 
Substituting the value of $h$ from Eq. (2.1.1) into Eq. (2.1.2),

$h_{a}=\frac{3(b+a) t-h_{b}(2 b+a)}{(b+2 a)}$

For the disc of isotropic material, by substituting $a=$ $31.75 \mathrm{~mm}, b=152.4 \mathrm{~mm}, t=1 \mathrm{~mm}$, and $h_{b}=1.78 \mathrm{~mm}$ in Eq. (2.1.3), we get the disc thickness at the inner radii $h_{a}=$ $0.55 \mathrm{~mm}$.

Using expression (2.1.3), Eq. (2.3) becomes

$$
\begin{aligned}
& A=(r-a)\left[h_{b}+c(2 b-r-a)\right] \\
& A_{0}=(b-a)\left[h_{b}+c(b-a)\right] \\
& I=\frac{h_{b}}{3}\left(r^{3}-a^{3}\right)+\frac{2 c b}{3}\left(r^{3}-a^{3}\right)-\frac{c}{2}\left(r^{4}-a^{4}\right) \\
& I_{0}=\frac{h_{b}}{3}\left(b^{3}-a^{3}\right)+\frac{2 c b}{3}\left(b^{3}-a^{3}\right)-\frac{c}{2}\left(b^{4}-a^{4}\right)
\end{aligned}
$$

\section{Dises with Hyperbolic Varying Thickness}

The thickness $(h)$ of the composite disc having hyperbolically varying thickness is assumed to be of the form

$h=c r^{m}$,

where $c$ and $m=-0.74$ are constants.

Since the volume of disc having hyperbolically varying thickness is assumed equal to the volume of constant thickness disc, therefore,

$c=\frac{(m+2)\left(b^{2}-a^{2}\right) t}{2\left(b^{m}+2-a^{m}+2\right)}$

For the disc of isotropic material, by substituting $a=$ $31.75 \mathrm{~mm}, b=152.4 \mathrm{~mm}, t=1 \mathrm{~mm}, m=-0.74$ in Eq. (2.2.2), we get $c=31.26$, and then, by using the value of $c$ in Eq. (2.2.1), the disc thickness at the inner radii and the outer radii are obtained respectively as $h_{a}=2.42 \mathrm{~mm}$ and $h_{b}=$ $0.76 \mathrm{~mm}$.

Using the expression for thickness (2.2.2), the Eq. (2.3) becomes

$$
\begin{aligned}
& A=c\left(\frac{r^{m+1}-a^{m+1}}{m+1}\right) \\
& A_{0}=c\left(\frac{b^{m+1}-a^{m+1}}{m+1}\right) \\
& I=c\left(\frac{r^{m+3}-a^{m+3}}{m+3}\right) \\
& I_{0}=c\left(\frac{b^{m+3}-a^{m+3}}{m+3}\right)
\end{aligned}
$$

\section{Distribution of Dispersoids and Creep Constants}

The distribution of silicon carbide particles in functionally graded material disc with variation in thickness, $h(r)$, is assumed to decrease linearly from inner to outer radius. Therefore, the density $\rho$ and the creep parameters of the disc will vary with radial distance. The composition variation in terms of volume percent of silicon carbide, along the radial distance, $V(r)$, is given as

$V(r)=V_{\max }-\frac{(r-a)}{(b-a)}\left(V_{\max }-V_{\min }\right)$
$V(r)=A-B r, \quad a \leq r \leq b$,

where

$A=V_{\max }+\mathrm{a} B$

and

$B=\frac{V_{\max }-V_{\min }}{b^{2}-a^{2}}$,

where $V_{\max }$ and $V_{\min }$ are the maximum and minimum particle contents, respectively, at the inner and outer radii of the disc.

According to law of mixture, the density variation $\rho(r)$ in the composite disc may be expressed as

$\rho(r)=\rho_{m}+\left(\rho_{d}-\rho_{m}\right) \frac{V(r)}{100}$,

where $\rho_{m}$ and $\rho_{d}$ are the densities of the aluminum based matrix and of the dispersed silicon carbide particles, respectively.

Substituting the value of $V(r)$ from Eq. (3.2) into Eq. (3.5),

$\rho(r)=\rho_{m}+\left(\rho_{d}-\rho_{m}\right) \frac{A-B r}{100}=A_{\rho}-B_{\rho} . r$,

where

$A_{\rho}=\rho_{m}+\left(\rho_{d}-\rho_{m}\right) \frac{A}{100}$

and

$B_{\rho}=\left(\rho_{d}-\rho_{m}\right) \frac{B}{100}$

The average particle content in the FGM disc $\left(V_{\text {avg }}\right)$ may be expressed as

$V_{\mathrm{avg}}=\frac{\int_{a}^{b} 2 \cdot \pi \cdot r \cdot h(r) \cdot V(r) d}{t \cdot\left(b^{2}-a^{2}\right) \cdot \pi}$ 
If the FGM disc is assumed to have constant thickness, then $V_{\text {min }}$ may be obtained by substituting the expression of $V(r)$ from Eq. (3.1) into Eq. (3.9),
For FGM disc with linear thickness, $V_{\min }$ may be obtained by substituting the expressions of $h(r)$ and $V(r)$ from Eqs. (2.1.1) and (3.1) into Eq. (3.9),

$V_{\min }=\frac{V_{\max }\left(b^{2}+a b-2 a^{2}\right)-V_{\mathrm{avg}}\left(b^{2}-a^{2}\right)}{\left(a^{2}+a b-2 b^{2}\right)}$

$V_{\min }=\frac{V_{\max }\left(\left(b^{2}+a b-2 a^{2}\right) h_{b}+c\left(3 a^{3}+a b^{2}+b^{3}-5 a^{2} b c\right)\right)-3 t V_{\mathrm{avg}}\left(b^{2}-a^{2}\right)}{\left(a^{2}+a b-2 b^{2}\right) h_{b}+c\left(a b^{2}+a^{2} b-a^{3}-b^{3}\right)}$,

where

$t=\frac{(b+2 a) h_{a}+h_{b}(2 b+a)}{3(b+a)}$,

where $t$ denotes the thickness of uniform thickness disc.

For FGM disc with hyperbolic thickness, $V_{\min }$ may be obtained by substituting the expressions of $h(r)$ and $V(r)$ from Eqs. (2.2.1) and (3.1) into Eq. (3.9)

$V_{\min }=\frac{V_{\max }\left[a^{m+2}(b(m+3)-a(m+2))-b^{m+3}\right]+\frac{t}{2 c} V_{\mathrm{avg}}\left(b^{2}-a^{2}\right)(b-a)(m+2)(m+3)}{b^{m+2}(b(m+2)-a(m+3))+a^{m+3}}$,

where

$t=\frac{2\left(b^{m+2}-a^{m+2}\right)}{(m+2)\left(b^{2}-a^{2}\right)} c$

\section{Mathematical Formulation of Creep in FGM Disc}

The present analysis assumes that the material of FGM disc is isotropic and yields according to von Mises criterion [22],

$f\left(\sigma_{i j}\right)=J_{2}-k^{2}=0$,

where $f\left(\sigma_{i j}\right)$ is the potential function, $k$ is a constant, and $J_{2}$ is given by

$J_{2}=\frac{1}{6}\left[\left(\sigma_{1}-\sigma_{2}\right)^{2}+\left(\sigma_{2}-\sigma_{3}\right)^{2}+\left(\sigma_{3}-\sigma_{1}\right)^{2}\right]$

The strain increment $\left(d \varepsilon_{i j}\right)$ is related to the potential function, $f\left(\sigma_{i j}\right)$, through the associated flow rule as given below.

$d \varepsilon_{i j}=d \lambda \frac{\partial f\left(\sigma_{i j}\right)}{\partial \sigma_{i j}}$ where $d \lambda$ is a proportionality factor that depends on $\sigma_{i j}$, $d \sigma_{i j}$, and $\varepsilon_{i j}$ apart from strain history because of strain hardening.

Substituting the yield criterion given by Eq. (4.1) into Eq. (4.3), the following constitutive equations are obtained in terms of principal strain increments $d \varepsilon_{11}, d \varepsilon_{22}$, and $d \varepsilon_{33}$ and principal stresses $\sigma_{11}, \sigma_{22}$, and $\sigma_{33}$.

$d \varepsilon_{11}=\frac{2}{3}\left[\sigma_{11}-\frac{\left(\sigma_{22}+\sigma_{33}\right)}{2}\right] d \lambda$
$d \varepsilon_{22}=\frac{2}{3}\left[\sigma_{22}-\frac{\left(\sigma_{11}+\sigma_{33}\right)}{2}\right] d \lambda$
$d \varepsilon_{33}=\frac{2}{3}\left[\sigma_{33}-\frac{\left(\sigma_{11}+\sigma_{22}\right)}{2}\right] d \lambda$ by

$\bar{\sigma}=\frac{1}{\sqrt{2}}\left[\left(\sigma_{22}-\sigma_{33}\right)^{2}+\left(\sigma_{33}-\sigma_{11}\right)^{2}+\left(\sigma_{11}-\sigma_{22}\right)^{2}\right]^{1 / 2}$

$d \bar{\varepsilon}=\frac{\sqrt{2}}{3}\left[\left(d \varepsilon_{1}-d \varepsilon_{2}\right)^{2}+\left(d \varepsilon_{2}-d \varepsilon_{3}\right)^{2}+\left(d \varepsilon_{3}-d \varepsilon_{1}\right)^{2}\right]^{1 / 2}$

The effective strain increment can be evaluated by substituting the values of $d \varepsilon_{11}, d \varepsilon_{22}$, and $d \varepsilon_{33}$ from Eq. (4.4) into Eq. (4.6) and using Eq. (4.5), 
$d \bar{\varepsilon}=\frac{2}{3} \bar{\sigma} d \lambda$

Using the value of $d \bar{\varepsilon}$ from Eq. (4.6) into Eq. (4.7), one gets the strain increments

$d \varepsilon_{11}=\frac{d \bar{\varepsilon}}{\bar{\sigma}}\left[\sigma_{11}-\frac{\left(\sigma_{22}+\sigma_{33}\right)}{2}\right]$

$d \varepsilon_{22}=\frac{d \bar{\varepsilon}}{\bar{\sigma}}\left[\sigma_{22}-\frac{\left(\sigma_{11}+\sigma_{33}\right)}{2}\right]$

$d \varepsilon_{33}=\frac{d \bar{\varepsilon}}{\bar{\sigma}}\left[\sigma_{33}-\frac{\left(\sigma_{11}+\sigma_{22}\right)}{2}\right]$

Strain rate can be obtained by integrating the above set of Eq. (4.8).

$\dot{\varepsilon}_{11}=\frac{\dot{\bar{\varepsilon}}}{2 \bar{\sigma}}\left[2 \sigma_{11}-\left(\sigma_{22}+\sigma_{33}\right)\right]$

$\dot{\bar{\varepsilon}}_{22}=\frac{\dot{\bar{\varepsilon}}}{2 \bar{\sigma}}\left[2 \sigma_{22}-\left(\sigma_{11}+\sigma_{33}\right)\right]$

$\dot{\bar{\varepsilon}}_{33}=\frac{\dot{\bar{\varepsilon}}}{2 \bar{\sigma}}\left[2 \sigma_{33}-\left(\sigma_{11}+\sigma_{22}\right)\right]$

Taking reference frame along the principal directions of $r$, $\theta$, and $z$, the generalized constitutive equations from Eq. (4.9) for creep in an isotropic composite disc under multiaxial stress condition are given as

$$
\begin{aligned}
& \dot{\varepsilon}_{r}=\frac{\dot{\bar{\varepsilon}}}{2 \bar{\sigma}}\left[2 \sigma_{r}-\sigma_{\theta}\right] \\
& \dot{\varepsilon}_{\theta}=\frac{\dot{\bar{\varepsilon}}}{2 \bar{\sigma}}\left[2 \sigma_{\theta}-\sigma_{r}\right], \\
& \dot{\varepsilon}_{z}=\frac{\overline{\bar{\varepsilon}}}{2 \bar{\sigma}}\left[-\left(\sigma_{r}+\sigma_{\theta}\right)\right]
\end{aligned}
$$

where $\dot{\varepsilon}_{r}, \dot{\varepsilon}_{\theta}, \dot{\varepsilon}_{z}$, and $\sigma_{r}, \sigma_{\theta}, \sigma_{z}$ are the strain rates and the stresses respectively in the FGM disc with variable thickness along $r, \theta$, and $z$ directions. $\dot{\bar{\varepsilon}}$ is the effective strain rate, and $\bar{\sigma}$ is the effective stress. For biaxial state of stress $\left(\sigma_{r}, \sigma_{\theta}\right)$, the effective stress is

$\bar{\sigma}=\left\{\frac{1}{2}\left\{\sigma_{\theta}^{2}+\sigma_{r}^{2}+\left(\sigma_{r}-\sigma_{\theta}\right)^{2}\right\}\right\}^{1 / 2}$

Using Eqs. (4.1), (4.11), and (4.10), it can be rewritten as

$$
\begin{aligned}
& \dot{\varepsilon}_{r}=\frac{d u_{r}}{d r}=\frac{[2 x(r)-1]\left[M(r)\left(\bar{\sigma}-\sigma_{0}(r)\right)\right]^{8}}{2\left[x^{2}(r)-x(r)+1\right]^{1 / 2}} \\
& \dot{\varepsilon}_{\theta}=\frac{u_{r}}{r}=\frac{[2-x(r)]\left[M(r)\left(\bar{\sigma}-\sigma_{0}(r)\right)\right]^{8}}{2\left[x^{2}(r)-x(r)+1\right]^{1 / 2}}
\end{aligned}
$$

From the material's incompressibility assumption, it follows that

$\dot{\varepsilon}_{z}=-\left(\dot{\varepsilon}_{r}+\dot{\varepsilon}_{\theta}\right)$,

where $x(r)=\frac{\sigma_{r}}{\sigma_{\theta}}$ is the ratio of radial and tangential stresses.

Dividing Eq. (4.12) by Eq. (4.13) and integrating the resulting equation by taking limit $a$ to $r$ on both sides,

$\dot{u}_{r}=\dot{u}_{r_{i}} \exp \int_{a}^{r} \frac{\varphi(r)}{r} d r$,

where $\dot{u}_{r_{i}}$ is the radial deformation rate at the inner radius and $\varphi(r)=\frac{2 x(r)-1}{2-x(r)}$

Substituting $\dot{u}_{r}$ from Eq. (4.15) into Eq. (4.13) and simplify to get the tangential stress $\left(\sigma_{\theta}\right)$,

$\sigma_{\theta}=\frac{\left(\dot{u}_{r_{i}}\right)^{1 / n}}{M(r)} \psi_{1}(r)+\psi_{2}(r)$,

where

$\psi_{1}(r)=\frac{\psi(r)}{\left[x^{2}(r)-x(r)+1\right]^{1 / 2}}$,

$\psi_{2}(r)=\frac{\sigma_{0}(r)}{\left[x^{2}(r)-x(r)+1\right]^{1 / 2}}$,

and

$\psi(r)=\left\{\frac{2}{r} \cdot \frac{\left[x^{2}(r)-x(r)+1\right]^{1 / 2}}{[2-x(r)]} \exp \cdot \int_{a}^{r} \frac{\varphi(r) d r}{r}\right\}^{1 / n}$

The equation of equilibrium for a rotating disc with varying thickness can be written as

$\frac{d}{d r}\left(r h(r) \sigma_{r}\right)-h(r) \cdot \sigma_{\theta}+\rho(r) \omega^{2} r^{2} h(r)=0$,

where $\rho(r)$ is the density of FGM disc.

Integrating Eq. (4.20) within limits $a$ to $b$ and substituting the values of $\rho(r)$ and $h(r)$ from Eqs. (3.5) and (2.1.1), respectively,

$\sigma_{\theta_{\text {avg }}}=\frac{\omega^{2}}{A_{0}}\left[A_{\rho} I_{0}-B_{\rho}\left(\frac{\left(h_{b}+2 b c\right)\left(b^{4}-a^{4}\right)}{4}-\frac{2 c\left(b^{5}-a^{5}\right)}{5}\right)\right]$,

where $\sigma_{\theta_{\text {avg }}}$ is the average tangential stress of FGM disc with linearly varying thickness. 
Fig. 2 Numerical scheme of computation

\section{LEGENDS:}

ITE $R=$ Ite ration no

$h=$ Limiting value of $\operatorname{Err}(=0.01)$

ITM $=$ Maximum no of iterations

$\mathrm{ERR}=\frac{\left[\sigma_{\theta}(r)\right]_{\mathrm{TTER}}-\left[\sigma_{\theta}(r)\right]_{\mathrm{TTER}-1}}{[\sigma]_{\mathrm{TTER}-1}}$

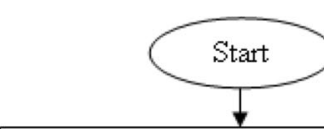

Enter values of $a, b, n, \omega, p$ and $t$

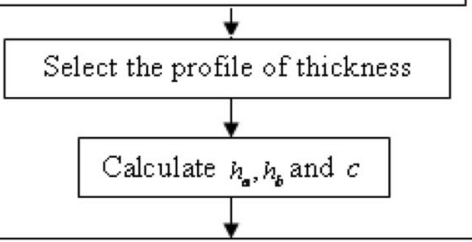

Calculate $V(r), \rho(r), M(r)$ and $\sigma_{0}(r)$

at differ ent points of the radius grid

$\checkmark$

Calculate $I_{0}, I, A, A_{0}$ and $\sigma_{\theta_{\max }}$

$\checkmark$

Initialization $\sigma_{\theta}=\sigma_{\theta \sigma \bar{x}}$

1

$\mathrm{TER}=1$

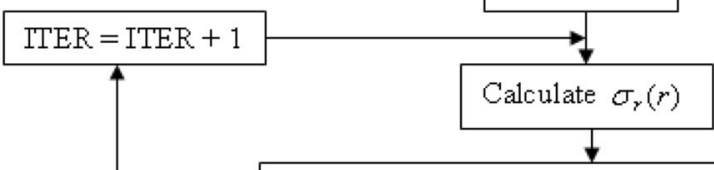

Calculate $x(r), \phi(r), \psi(r), \psi_{1}(r), \psi_{2}(r)$ and $\dot{u}_{a}{ }^{1 / m}$

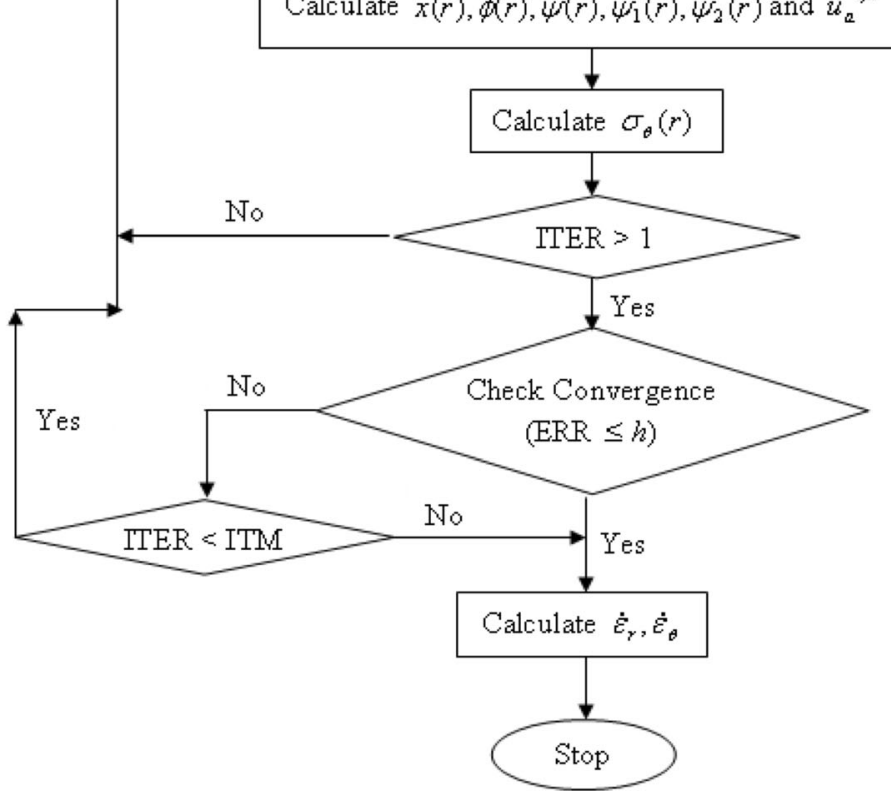

For FGM disc with constant thickness, the average tangential stress can be obtained by substituting $h_{b}=1$ and $c=0$ in Eq. (4.21),

$\sigma_{\theta_{\mathrm{avg}}}=\frac{\omega^{2}}{(b-a)}\left[A_{\rho} \frac{\left(b^{3}-a^{3}\right)}{3}-B_{\rho} \frac{\left(b^{4}-a^{4}\right)}{4}\right]$
Integrating Eq. (4.20) within limits $a$ to $b$ and substituting the values of $\rho(r)$ and $h(r)$ from Eqs. (3.5) and (2.2.1), respectively,

$$
\sigma_{\theta_{\mathrm{avg}}}=\frac{\omega^{2}}{A_{0}}\left[A_{\rho} I_{0}-B_{\rho} \cdot c \cdot\left(\frac{b^{m+4}-a^{m+4}}{m+4}\right)\right] \text {, }
$$


Table 2 Values of Wahl et al. [23] experimental data

Parameters for steel disc:

Density of disc material $\rho=2862.1 \mathrm{~kg} / \mathrm{m}^{3}$

Inner radius of disc, $a=31.75 \mathrm{~mm}$

Outer radius of disc, $b=152.4 \mathrm{~mm}$

Particle size, $P=1.7 \mu \mathrm{m}$

Uniformly distributed particle content, $V_{\text {avg }}=20 \%$

For FGM disc with constant thickness, $V_{\max }=35 \%$ and $V_{\min }=$ $10.38 \%$

For FGM disc with linearly varying thickness, $V_{\max }=35 \%$ and $V_{\min }=$ $7.55 \%$

For FGM disc with hyperbolically varying thickness, $V_{\max }=35 \%$ and $V_{\min }=6.74 \%$

For non-FGM disc creep parameters: $M(r)=6.16 \times 10^{-3} \mathrm{~s}^{-1 / 8} / \mathrm{MPa}$ and $\sigma_{0}(r)=24.51 \mathrm{MPa}$

Density, $\mathrm{Al}=2713 \mathrm{~kg} / \mathrm{m}^{3}$

Density, $\mathrm{SiC}=3210 \mathrm{~kg} / \mathrm{m}^{3}$

The disc thickness for constant thickness at the inner radii, $h_{a}=1 \mathrm{~mm}$ The disc thickness for constant thickness at the outer radii, $h_{b}=1 \mathrm{~mm}$ The disc thickness for linearly varying thickness at the inner radii, $h_{a}=$ $1.44 \mathrm{~mm}$

The disc thickness for linearly varying thickness at the outer radii, $h_{b}=$ $0.75 \mathrm{~mm}$

The disc thickness for hyperbolically varying thickness at the inner radii, $h_{a}=2.42 \mathrm{~mm}$

The disc thickness for hyperbolically varying thickness at the outer radii, $h_{b}=0.76 \mathrm{~mm}$

Operating conditions:

Angular velocity of disc, $\omega=15,000 \mathrm{rpm}$

Operating temperature, $T=623 \mathrm{~K}$ where $\sigma_{\theta_{\text {avg }}}$ is the average tangential stress of FGM disc with hyperbolically varying thickness.

Substituting $\sigma_{\theta}$ from Eq. (4.16) into (2.4),

$$
\left(\dot{u}_{r_{i}}\right)^{1 / n}=\frac{A_{0} \sigma_{\theta_{\text {avg }}}-\int_{a}^{b} \psi_{2}(r) \cdot h(r) d r}{\int_{a}^{b} \frac{\psi_{1}(r) \cdot h(r)}{M(r)} d r}
$$

Using Eqs. (4.24) and (4.16), it becomes

$$
\sigma_{\theta}=\frac{\psi_{1}(r)\left[A_{0} \sigma_{\theta_{\text {avg }}}-\int_{a}^{b} \psi_{2}(r) \cdot h(r) d r\right]}{M(r) \int_{a}^{b} \frac{\psi_{1}(r) \cdot h(r)}{M(r)} d r}+\psi_{2}(r)
$$

Integrating Eq. (4.20) within limits $a$ to $r$ and substituting the values of $\rho(r)$ and $h(r)$ from Eqs. (3.5) and (2.1.1), respectively,

$$
\sigma_{r}=\frac{1}{r \cdot h(r)}\left[\begin{array}{l}
\int_{a}^{r} \sigma_{\theta} \cdot h(r) d r-\omega^{2} A_{\rho} I+\omega^{2} B_{\rho} \\
\left(\frac{\left(h_{b}+2 b c\right)\left(r^{4}-a^{4}\right)}{4}-\frac{2 c\left(r^{5}-a^{5}\right)}{5}\right)
\end{array}\right],
$$

Fig. 3 Variation of particle content in non-FGM and FGM composite discs

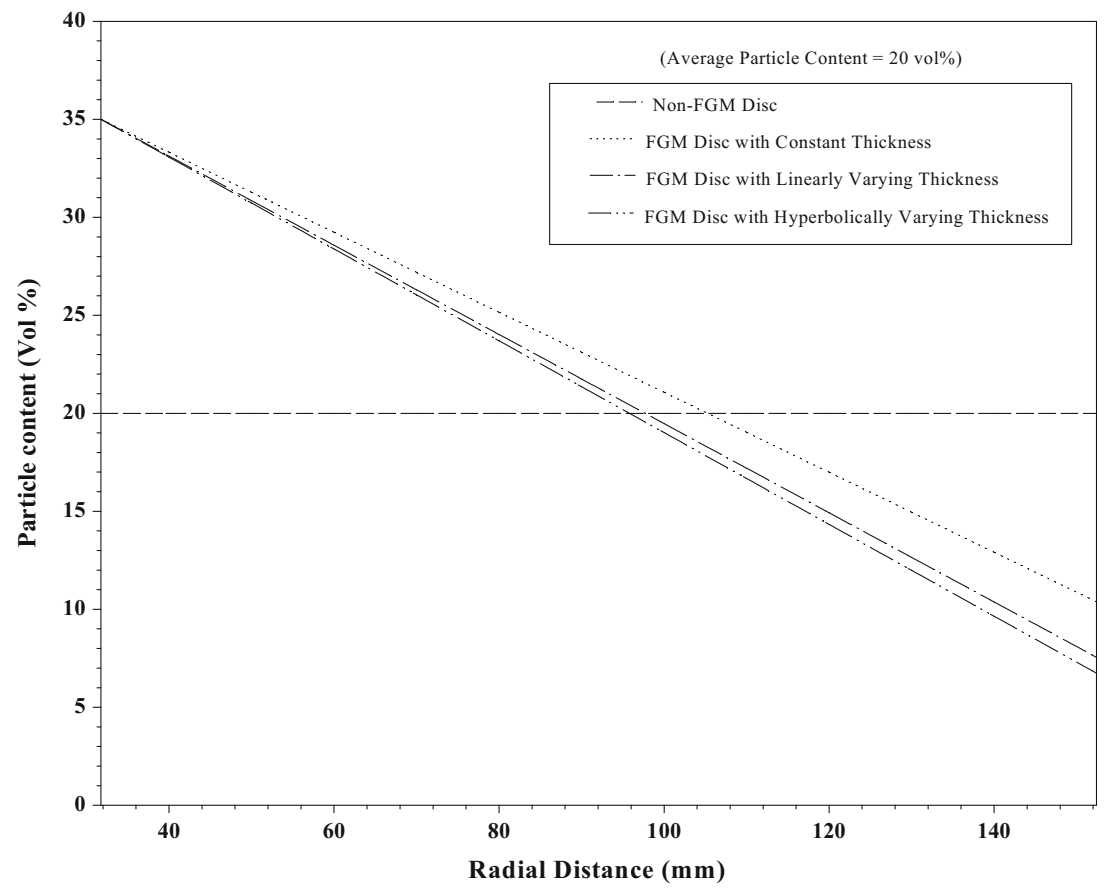


Fig. 4 Variation of creep parameter in non-FGM and FGM composite discs

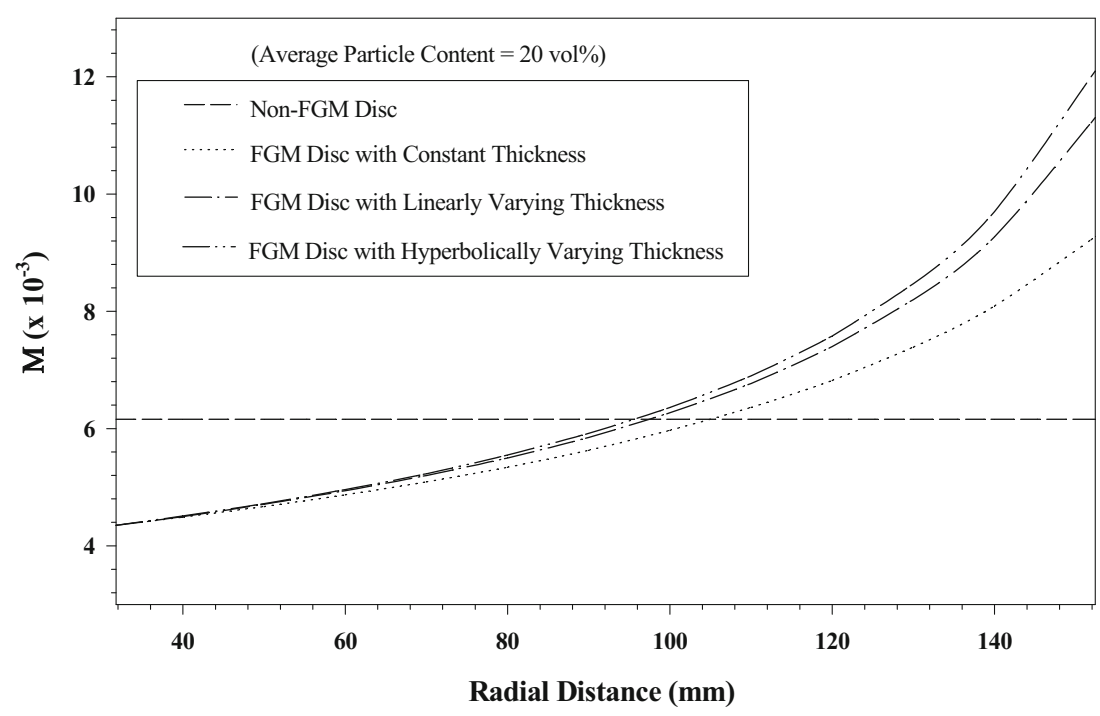

where $\sigma_{r}$ be the radial stress of FGM disc with linearly varying thickness.

For FGM disc with constant thickness, the radial stress can be obtained by substituting $h_{b}=1$ and $c=0$ in Eq. (4.26),

$\sigma_{r}=\frac{1}{r}\left[\int_{a}^{r} \sigma_{\theta} d r-\omega^{2} A_{\rho} \frac{\left(r^{3}-a^{3}\right)}{3}+\omega^{2} B_{\rho} \frac{\left(r^{4}-a^{4}\right)}{4}\right]$

Integrating Eq. (4.20) within limits $a$ to $b$ and substituting the values of $\rho(r)$ and $h(r)$ from Eqs. (4.5) and (2.2.1), respectively,

$\sigma_{r}=\frac{1}{r \cdot h(r)}\left[\int_{a}^{r} \sigma_{\theta} \cdot h(r) d r-\omega^{2} A_{\rho} I+\omega^{2} B_{\rho} c \cdot\left(\frac{r^{m+4}-a^{m+4}}{m+4}\right)\right]$,

where $\sigma_{r}$ is the radial stress of FGM disc with hyperbolically varying thickness.
Thus, for FGM disc with constant thickness, the tangential stress $\sigma_{\theta}$ and radial stress $\sigma_{r}$ are determined by Eqs. (4.22) and (4.27), respectively. For FGM disc with linearly varying thickness, the tangential stress $\sigma_{\theta}$ and radial stress $\sigma_{r}$ are determined by Eqs. (4.21) and (4.26), respectively. For FGM disc with hyperbolically varying thickness, the tangential stress $\sigma_{\theta}$ and radial stress $\sigma_{r}$ are determined by Eqs. (4.23) and (4.28), respectively. Then, strain rates $\varepsilon_{r}, \varepsilon_{\theta}$, and $\varepsilon_{z}$ are calculated from Eqs. (4.12), (4.13), and (4.14).

\section{Numerical Computations}

Following the procedure described in previous sections, the distributions of stress and strain rate are estimated through an iterative numerical scheme of computation shown in Fig. 2. The iteration is continued till the process converges and gives the values of stresses at different points of the radius grid. For rapid convergence, $75 \%$ of the value of $\sigma_{\theta}$ obtained in the
Fig. 5 Variation of creep parameter in non-FGM and FGM composite discs

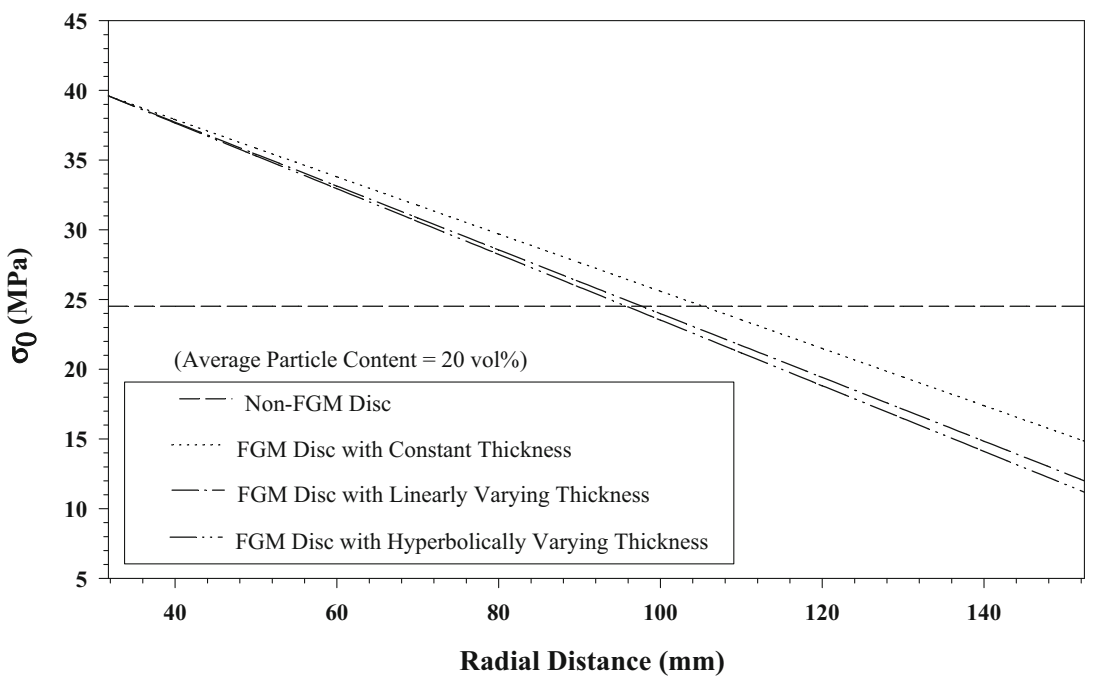



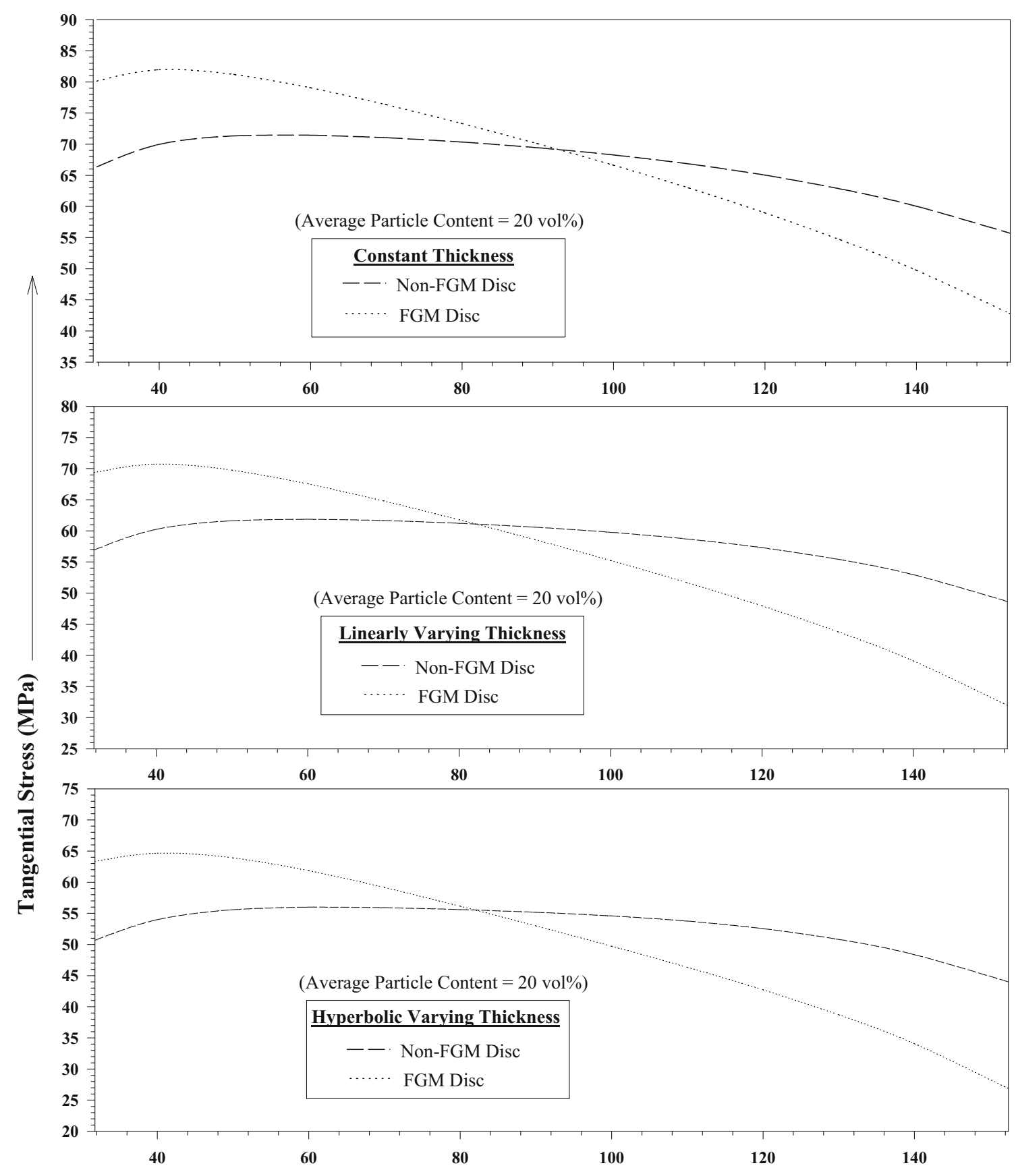

Radial Distance (mm)

Fig. 6 Variation of tangential stress along the radial distance in the isotropic non-FGM and FGM composite discs rotating with an angular velocity $15,000 \mathrm{rpm}$ at $623 \mathrm{~K}$

current iteration has been mixed with $25 \%$ of the value of $\sigma_{\theta}$ obtained in the last iteration for use in the next iteration, i.e., $\sigma_{\theta \text { next }}=0.25 \sigma_{\theta \text { previous }}+0.75 \sigma_{\theta \text { current }}$.

\section{Results and Discussion}

A computer code based on the mathematical formulation has been developed to obtain the creep response of the rotating composite variable thickness (constant, linear, and hyperbolic) discs (i) with uniform particle content (non-FGM disc) and (ii) with particle gradient (FGM disc). Results for these two discs for each profile have been compared under a constant temperature. For all the FGM discs, maximum particle content, $V_{\max }$, is $35 \%$, and $V_{\min }$ is estimated from Eqs. (3.10), (3.11), and (3.12) according to thickness profile. After this, the content of silicon carbide particle, $V(r)$, in the different FGM discs is calculated from Eq. (3.1). Thus, obtained values of $V(r)$, for 

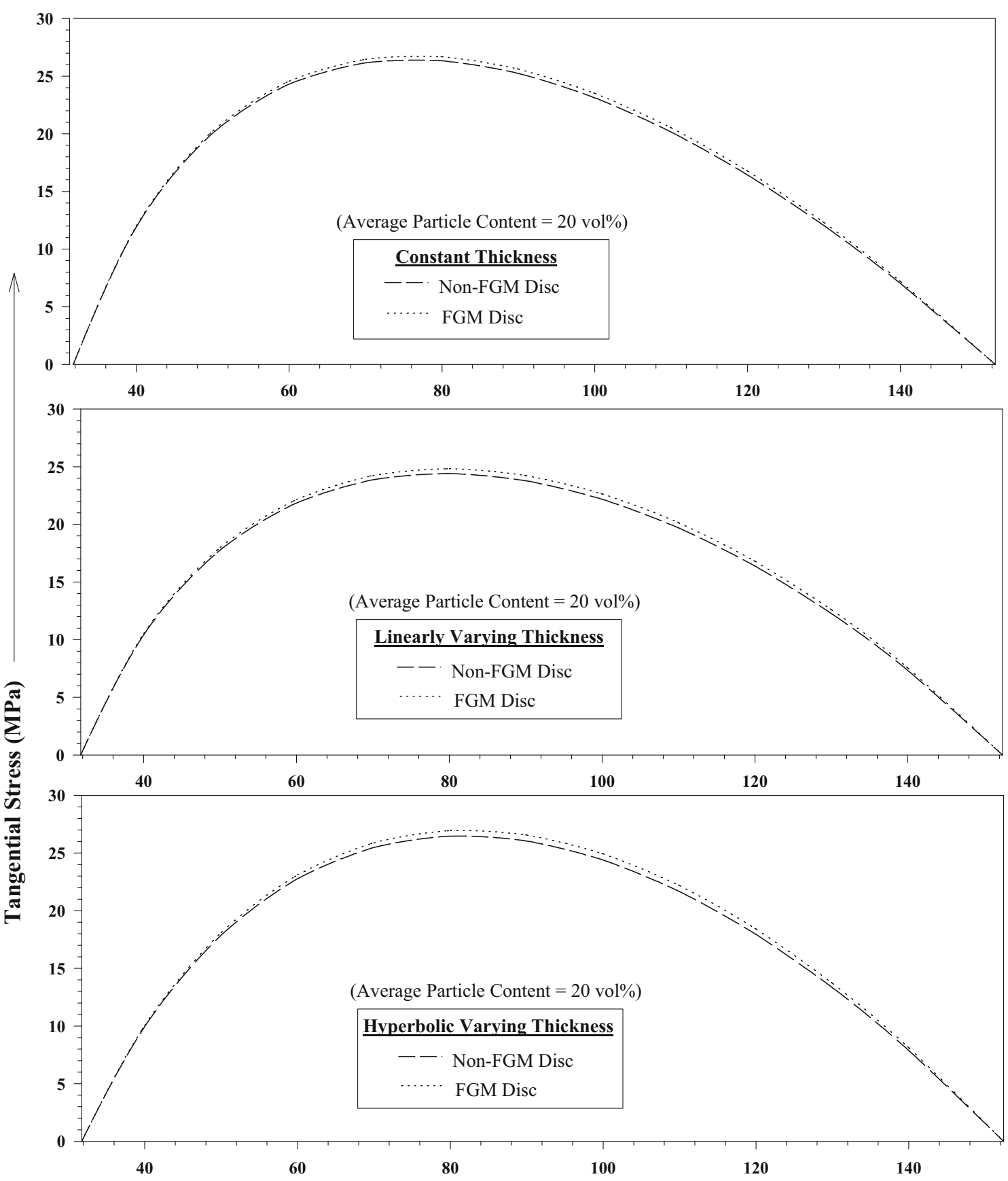

Radial Distance (mm)

Fig. 7 Variation of radial stress along the radial distance in the isotropic non-FGM and FGM composite discs rotating with an angular velocity $15,000 \mathrm{rpm}$ at $623 \mathrm{~K}$

all FGM discs with thickness variation, are substituted to get the variation of the creep parameters, $M(r)$ and $\sigma_{0}(r)$, in each FGM discs. To obtain the plastic stresses and strain rates, the results for a rotating steel disc by following the current analysis scheme were obtained for disc and operating conditions for which are mentioned in Table 2.

Before discussing the results of the steady-state creep response obtained in this study, it is considered necessary to validate the analysis carried out and the software developed. To achieve this goal, the results for a rotating composite disc by following the current analysis scheme were obtained for the disc with operating conditions for which are mentioned in Table 2.

Figure 3 shows the distribution of reinforcement in various composite discs. The particle content $V(r)$ in FGM disc with thickness variation (constant, linear, and hyperbolic) decreases linearly from the inner to the outer radius, and in 


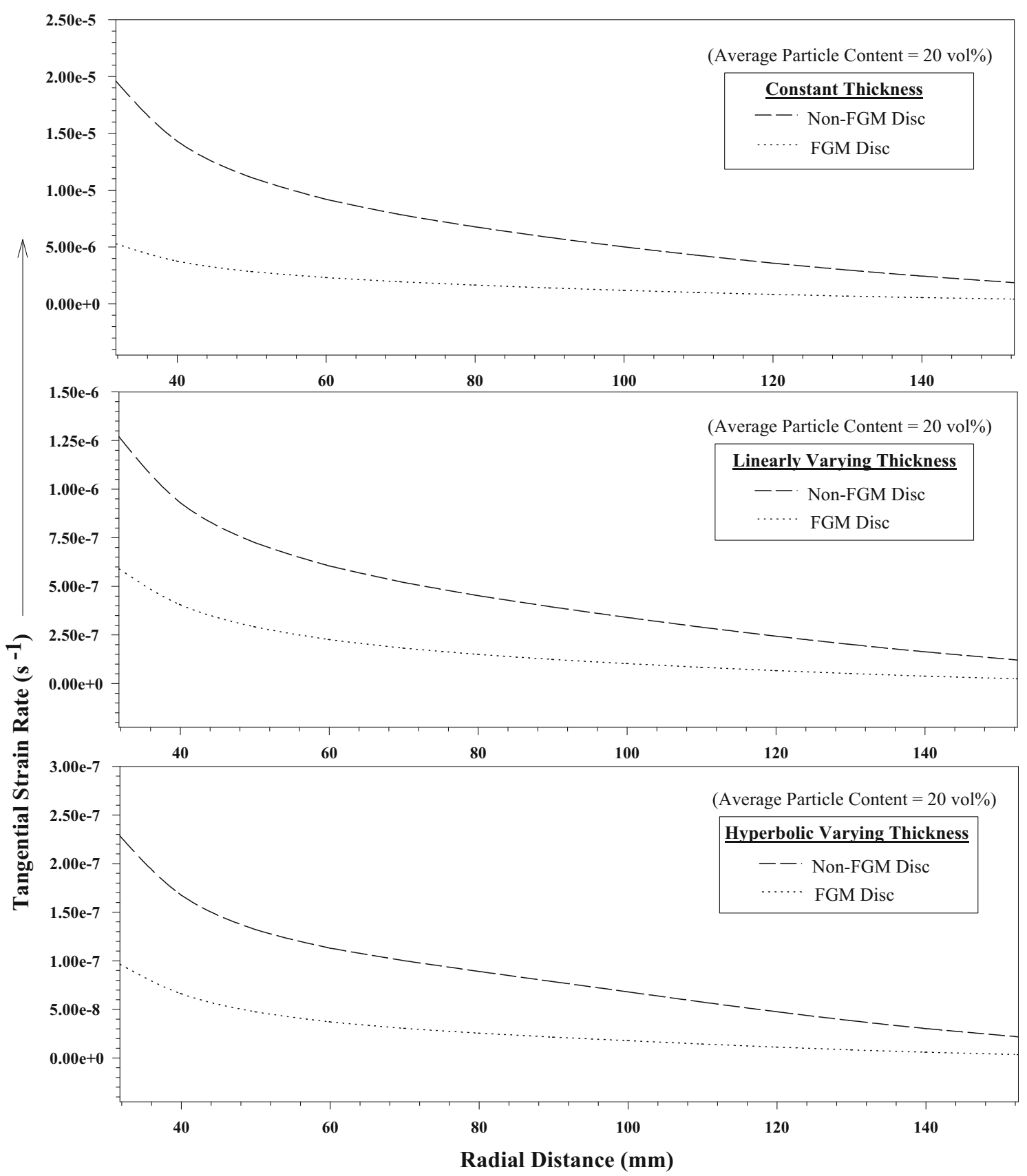

Fig. 8 Variation of tangential strain rate along the radial distance in the isotropic non-FGM and FGM composite discs rotating with an angular velocity $15,000 \mathrm{rpm}$ at $623 \mathrm{~K}$

non-FGM disc, the particle content is uniformly 20 vol.\% over the entire radius, although the variation of $V(r)$ becomes steeper by choosing the FGM disc with hyperbolic thickness.

Figures 4 and 5 show the variation of material parameters $M(r)$ and $\sigma_{0}(r)$, respectively, along radial distance for the different (FGM and non-FGM) composite discs. Both the material parameters, $M(r)$ and $\sigma_{0}(r)$, were observed to be constant over the entire non-FGM disc due to uniform distribution of particle content of silicon carbide (20 vol. \%) in aluminum matrix. But in FGM disc, the creep parameter $M(r)$ increases with increasing radial distance due to a decrease in particle content $V(r)$ on moving from the inner to the outer radius, as evident from Eq. (3.2). At the inner disc in FGM disc with thickness variation (constant, linear, and hyperbolic), the value of creep parameter $M(r)$ is the same (i.e., $4.35 \times 10^{-3} \mathrm{~s}^{-1 / 8} / \mathrm{MPa}$ ), but near the outer radius in FGM disc with hyperbolic thickness, creep parameter $M(r)$ has maximum value (i.e., $1.21 \times 10^{-2} \mathrm{~s}^{-1 / 8} / \mathrm{MPa}$ ) than those of FGM disc with thickness variation (constant and linear). On the other side, the threshold stress $\sigma_{0}(r)$ in FGM disc decreases linearly on moving from the inner to the outer radius. The threshold stress is maximum and the same at the inner radius in FGM disc with each thickness profile (i.e., $24.51 \mathrm{MPa}$ ) because of 


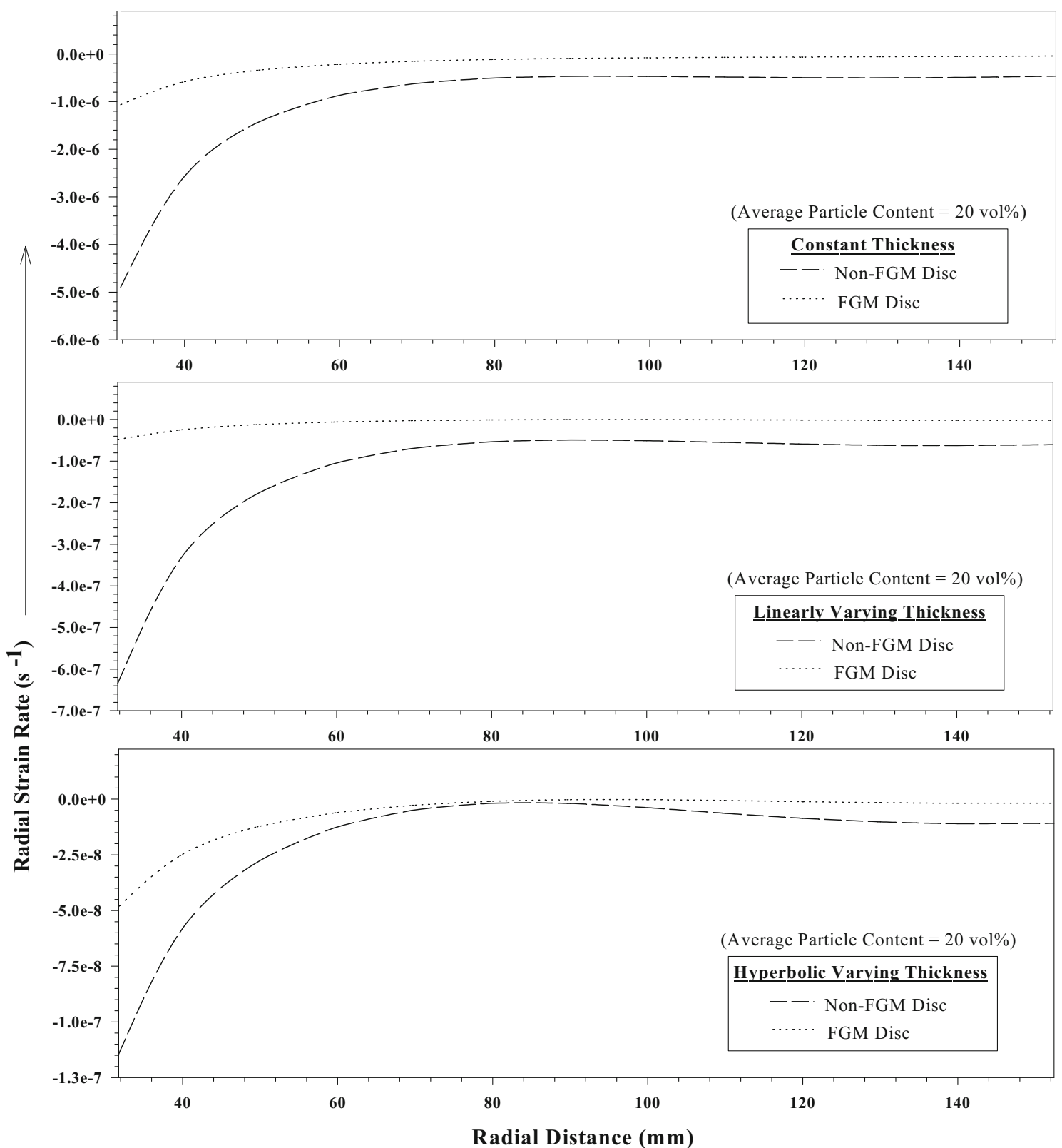

Fig. 9 Variation of radial strain rate along the radial distance in the isotropic non-FGM and FGM composite discs rotating with an angular velocity $15,000 \mathrm{rpm}$ at $623 \mathrm{~K}$

more amount of particle content of silicon carbide shown in Fig. 5, but near the outer radius by choosing FGM disc with hyperbolic thickness, the threshold stress is minimum (i.e., 11.18 MPa) than those of FGM disc with constant and linear thickness profile.

Figures 6, 7,8, and 9 show the steady-state creep behavior of the non-FGM and FGM discs with constant and varying thickness. The material of all the composite discs (non-FGM disc and FGM) is isotropic. It is observed that the tangential stress in all the composite discs increases with increasing radial distance, reaches maximum, and then decreases on moving towards the outer radius of the discs as shown in Fig. 6. The FGM discs with thickness variation (constant, linear, and hyperbolic), which having linearly decreasing particle content from the inner to outer radius, have a relatively higher tangential stress near the inner radius but lower values of tangential stress near the outer radius than that observed in non-FGM discs with thickness variation (constant, linear, and hyperbolic). The primary reason for these changes in the tangential stress distribution is the variation of density in the FGM disc. The higher density near the inner radius due to the higher amount of particle content and relatively lower density near the outer radius due to small amount of particle content have resulted in higher and lower tangential stress, respectively, compared to tangential stress in the non-FGM disc having the average density due to uniform distribution of particles, as shown in Fig. 6. The trend of 
variation of tangential stress is similar in all cases of thickness profile variations of FGM/non-FGM disc. The tangential stress in isotropic rotating FGM/non-FGM disc having hyperbolic thickness is reduced everywhere compared to FGM/non-FGM disc of uniform and linear thickness.

The radial stress increases from zero to reach a maximum value in the middle of the non-FGM and FGM disc followed by a decrease to reach at zero level again at the outer radius, under the imposed boundary conditions of vanishing radial stress at the inner and the outer radii of the disc, as shown in Fig. 7. The radial stress is the highest over the entire disc in the FGM disc with each thickness profile, having linear distribution of particle content along the radial distance, and the lowest in the non-FGM disc with thickness variation (constant, linear, and hyperbolic) which has uniform distribution of particle content. In all the FGM/non-FGM discs with each thickness variations, the tangential strain rate is the highest at the inner disc and then decreases continuously when one moves towards the outer disc. In the FGM/non-FGM disc with hyperbolic variation of thickness, the tangential strain rate is relatively less compared to the strain rate in other two variations in the thickness of the FGM/nonFGM disc. On the other hand, the steady-state tangential strain rate developing in a rotating FGM varying thickness disc with linearly decreasing particle distribution from inner to outer radius is lower over the entire disc as compared to those in a non-FGM varying thickness disc with uniform particle distribution. In the region near the inner radius, the tangential strain rate decreases due to higher particle content whereas near the outer radius, the strain rate decreases due to lower tangential stress in spite of lower particle content, resulting due to lower density of the FGM varying thickness discs towards the outer radius. Thus, the combination of FGM disc with hyperbolic thickness yields less tangential strain rate. The compressive radial strain rate is at maximum in the inner radius and goes on decreasing up to a certain radial distance followed by an increasing trend towards the outer radius in all the composite discs. The distribution of radial strain rate becomes relatively more uniform in the FGM disc in comparison to the non-FGM disc. This uniformity in distribution is due to the varying particle content in the FGM disc. Therefore, the FGM disc with hyperbolic thickness will have less chances of distortion.

\section{Conclusion}

Based on the above results and discussion, the following main conclusions may be drawn:

1. The steady-state creep rates in the FGM varying thickness disc with linearly decreasing particle content are significantly lower than that observed in a similar profile disc with the same particle content distributed uniformly. The decrease observed in tangential strain rate in the FGM varying thickness disc with linearly decreasing particle content is more at the inner radius than that observed towards the outer radius.

2. The magnitude of tangential and radial stresses in the FGM disc with linearly decreasing particle content is reduced significantly, when the disc thickness profile changes from uniform to hyperbolic.

3. By employing isotropic rotating FGM disc with hyperbolic thickness, the distribution of strain rates becomes relatively uniform as compared to a similar FGM disc with constant and linear thickness, which may reduce the chances of distortion in the FGM disc.

4. In an isotropic rotating FGM hyperbolic disc with linearly decreasing particle content from the inner to the outer radius, the steady-state creep response is significantly superior compared to that in the same thickness profile disc with the same particle content distributed uniformly.

\section{References}

1. Bayat MN, Saleem M, Sahari BB, Hamouda AMS, Mahdi E. Thermo elastic analysis of a functionally graded rotating disc with small and large deflections. Thin-Walled Struct. 2007;45(7-8):677-91.

2. Bektas NB, Acka M. Stress analysis of functionally graded discs under mechanical and thermal loads: analytical and numerical solutions. Sci Eng Compos Mater. 2012;19(4):361-71.

3. Bhatnagar NS, Kulkarni PS, Arya VK. Steady state creep of orthotropic rotating discs of variable thickness. Nucl Eng Des. 1986;91(2):121-41.

4. Chamoli N, Singh SB. Creep analysis of a functionally graded rotating disc in the presence of residual stress. Can J Mech Sci Eng. 2012;3(3):1-11.

5. Deepak D, Gupta VK, Dham AK. Investigating the effect of thickness profile of a rotating functionally graded disc on its creep behavior. J Thermoplast Compos Mater. 2013;26(4):461-75.

6. Garg M, Salaria BS, Gupta VK. Effect of reinforcement gradient on steady state creep in a variable thickness rotating disc made of nonlinear FGM. Int J Mater Eng Innov (IJMATEI). 2013;4(1):1-17.

7. Goel N, Garg M, Saini BS, Gupta VK. Finite element analysis of creep in a functionally graded rotating disc. Int J Comput Aid Eng Technol. 2012;4(5):432-44.

8. Gupta VK, Singh SB, Chandrawat HN, Ray S. Modeling of creep behaviour of a rotating disc in presence of both composition and thermal gradients. J Eng Mater Technol. 2005;127(1):97-105.

9. Jahed H, Farshi B, Bidabadi J. Minimum weight design of inhomogeneous rotating discs. Int J Press Vessel Pip. 2005;82(1):35-41.

10. Ma BM. Creep analysis of rotating solid discs. J Frankl Inst. 1959;267(2):149-65.

11. Ma BM. Creep analysis of rotating solid discs with variable thickness and temperature. J Frank1 Inst. 1961;271(1):40-55.

12. Ma BM. A power function creep analysis for rotating solid discs having variable thickness and temperature. J Franklin Inst. 1964;277(6):593-612.

13. Mishra RS, Pandey AB. Some observations on the hightemperature creep behaviour of $6061 \mathrm{Al}-\mathrm{SiC}$ composites. Metall Trans. 1990;21A(7):2089-91.

14. Pandey AB, Mishra RS, Mahajan YR. Steady state creep behavior of silicon carbide particulate reinforced aluminium composites. Acta Metall Mater. 1992;40(8):2045-52. 
15. Penny RK, Mariott DL. Design for creep. London: Chapman and Hall; 1995.

16. Rattan M, Singh SB, Ray S. Effect of stress exponent of steady state creep in an isotropic rotating disc. Bull Calcutta Math Soc. 2009;101(6):559-70.

17. Rattan M, Chamoli N, Singh SB. Creep analysis of an isotropic functionally graded rotating disc. Int J Contemp Math Sci. 2010;5(9):419-31.

18. Reddy JN, Wang CM, Kitipornchai S. Axisymmetric bending of functionally graded circular and annular plates. Eur J Mech A Solids. 1999;18(2):185-99.

19. Sharma S, Sahni M. Elastic-plastic analysis for finite deformation of a rotating disc having variable thickness with inclusion. World Acad Sci Eng Technol. 2011;5(3):336-46.
20. Sherby OD, Klundt RH, Miller AK. Flow stress, subgrain size and subgrain stability at elevated temperature. Metall Trans. 1977;8A: $843-50$.

21. Singh SB, Ray S. Steady-state creep behavior in an isotropic functionally graded material rotating disc of $\mathrm{Al}-\mathrm{SiC}$ composite. Metall Trans. 2001;32A(7):1679-85.

22. Von Mises R. Mechanics of solids in the plastically deformable state. NASA Tech Memo. 1913;88488:1986.

23. Wahl AM, Sankey GO, Manjoine MJ, Shoemaker E. Creep tests of rotating risks at elevated temperature and comparison with theory. $\mathrm{J}$ Appl Mech. 1954;76:225-35.

24. Zenkour AM. Elastic deformation of the rotating functionally graded annular disc with rigid casing. J Mater Sci. 2007;42(23):1-17. 